\title{
Expression of Lysine-specific demethylase 1 in human epithelial ovarian cancer
}

\author{
Cong Chen ${ }^{1+}$, Jing Ge ${ }^{2 \dagger}$, Qibin Lu', Guoqiang Ping ${ }^{3}$, Chunqing Yang ${ }^{3 *}$ and Xuefeng Fang ${ }^{4 *}$
}

\begin{abstract}
Background: Lysine-specific demethylase 1(LSD1) is implicated in the tumorigenesis and progression in various cancers. However, the expression of LSD1 in epithelial ovarian cancer and its clinical significance has not been examined in detail.

Methods: Immunohistochemical was used to detect the expression of LSD1 in normal ovarian epithelial tissues, cystadenoma, borderline cystadenoma, and cystadenocarcinoma. Next, the correlations between expression of LSD1 and clinicopathological features was assessed in 96 species of serous cystadenocarcinoma and 36 species of mucinous cystadenocarcinoma.
\end{abstract}

Results: Immunohistochemical results showed that the expression of LSD1 was gradually increased from benign cystadenoma and borderline cystadenoma to cystadenocarcinoma. The positive ratio of LSD1 expression was $50 \%$ in normal ovarian epithelial tissues, $72 \%$ in serous cystadenoma, $73 \%$ in mucinous cystadenoma, $82 \%$ in borderline serous cystadenoma, $83 \%$ in borderline mucinous cystadenoma, 94\% in serous cystadenocarcinoma and $92 \%$ in mucinous cystadenocarcinoma, respectively. LSD1 expression levels were associated with International Federation of Gynecology and Obstetrics stage and lymphatic metastasis in both serous and mucinous cystadenocarcinoma samples. Kaplan-Meier curves suggested that overall survival time of patients with high LSD1 expression was significantly shorter than that of patients with low LSD1 expression. Multivariate Cox proportional hazard regression indicated that higher LSD1 expression was a significant independent predictor of poor survival of EOC patients $(P=0.016)$.

Conclusions: These results suggest that LSD1 may be involved in carcinogenesis and progression with promising therapeutic potential for epithelial ovarian cancer.

Keywords: Epithelial ovarian cancer, Lysine-specific demethylase 1, Serous cystadenocarcinoma, Mucinous cystadenocarcinoma

\section{Introduction}

Ovarian cancer is the second most common gynecologic cancer and ranks the most lethal gynecological malignancy in the world, due to its high incidence of metastasis and high relapse rate [1]. The vast majority of ovarian cancer will be epithelial ovarian cancer (EOC), which comprises three major histological subtypes (serous, mucinous, and endometrioid).

\footnotetext{
* Correspondence: yangchunqingnj@163.com; xffang@zju.edu.cn ${ }^{\dagger}$ Equal contributors

${ }^{3}$ Department of Pathology, First Affiliated Hospital of Nanjing Medical University, Nanjing, Jiangsu 210029, China

${ }^{4}$ Department of Medical Oncology, Second Affiliated Hospital, Zhejiang

University College of Medicine, Hangzhou, Zhejiang 310009, China

Full list of author information is available at the end of the article
}

Unfortunately, the most of EOC patients were diagnosed at FIGO (International Federation of Gynecology and Obstetrics stage) III/IV stage and had unfavorable prognosis, with a frustrating 5-year overall survival $(<50 \%)$ [2]. Although the molecular alterations in EOC have been widely studied, studying the mechanism that regulates the initiation and progression will provide further insights into the development and progression of EOC.

Lysine-specific demethylase 1 (LSD1) is the first histone demethylase to be discovered [3]. Overexpression of LSD1 has also been associated with unfavourable

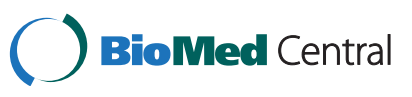

(C) 2015 Chen et al.; licensee BioMed Central. This is an Open Access article distributed under the terms of the Creative Commons Attribution License (http://creativecommons.org/licenses/by/4.0), which permits unrestricted use, distribution, and reproduction in any medium, provided the original work is properly credited. The Creative Commons Public Domain Dedication waiver (http://creativecommons.org/publicdomain/zero/1.0/) applies to the data made available in this article, unless otherwise stated. 
clinicopathological characteristics and poor prognosis in numerous tumors, such as hepatocellular carcinoma, colon cancer, breast cancer, prostate cancer, and non-small cell lung cancer [4-8]. Recently, one study showed that the expression of LSD1 mRNA was increased in ovarian tumors and LSD1 mRNA was overexpressed in stage IIIC and high-grade ovarian tumors [9]. However, the expression and significance of LSD1 protein in EOC is still poorly understood.

This study was to systematically investigate LSD1 protein expression in normal ovarian epithelium, benign cystadenoma, borderline cystadenoma, and cystadenocarcinoma using immunohistochemical (IHC) staining. The potential correlation between the expression level of LSD1 and clinicopathological features of EOC was also analyzed.

\section{Materials and methods Tissue specimens}

The specimens $(n=407)$ were randomly obtained from patients with complete clinical data in the Pathological Department of Jiangsu Province Hospital and Second Affiliated Hospital of Zhejiang University College of Medicine between March 2000 and May 2013. All patients were evaluated for histological type and graded by the two gynecological pathologists. The samples consisted of 50 normal ovarian epithelia species; 199 species of serous epithelial lesions, and 158 species of mucinous epithelial lesions. None of the patients were treated with chemotherapy, immunotherapy or radiotherapy prior to specimen collection. Tissue samples were obtained after patients' written informed consent under a general tissue collection protocol approved by The Research Ethics Committee of Jiangsu Province Hospital and The Research Ethics Committee of Second Affiliated Hospital of Zhejiang University College of Medicine.

\section{Immunohistochemistry}

The immunohistochemistry (IHC) for LSD1 (1:100; Cell Signaling Inc., Danvers, MA, USA) was performed on formalin-fixed, paraffin-embedded tissue sections using steam heatinduced epitope retrieval and the DAB chromogen (Boster, Wuhan, China). Positive cells were indicated by the presence of brown staining in the nucleus. The LSD1 expression was evaluated based on intensity of staining and distribution of positive cells. Both the percentage of positive cells and the staining intensity were evaluated under double-blind conditions. The percentage positivity was scored as four classes: "0" (<5\%, negative), "1" (5-25\%, sporadic), “2" (25-50\%, focal), or " 3 " (>50\%, diffuse). The staining intensity was scored as "0" (no staining), "1" (weakly stained), "2" (moderately stained), or " 3 " (strongly stained). The LSD1 immunostaining score was calculated as the percentage positive score $\times$ the staining intensity score and ranged as 0 , $1,2,3,4,6$, and 9. The sum-indexes $(-),(+),(++)$, and $(+++)$ indicated overall staining score of $0,1-3$, 4-6, and 9, respectively. For statistical analysis, sumindexes (-) and (+) were defined as low LSD1 expression, while sum-indexes $(++)$ and $(+++)$ were defined as high LSD1 expression.

\section{Statistical analysis}

Statistical analysis was carried out using SPSS version 16.0. LSD1 expression in different groups was analyzed using the non-parametric tests. The approximate normal distribution of the two groups is represented as Z-score. The correlation between LSD1 expression and the clinicopathological features was assessed by chi-square test. Survival analyses were performed using the log-rank test and Kaplan-Meier plots approach. Variables with $P$ value $<0.05$ in univariate analysis were used in thesubsequent multivariate analysis on a basis of Cox proportionalhazards model. For all analyses, the level of significance was set at $P<0.05$.

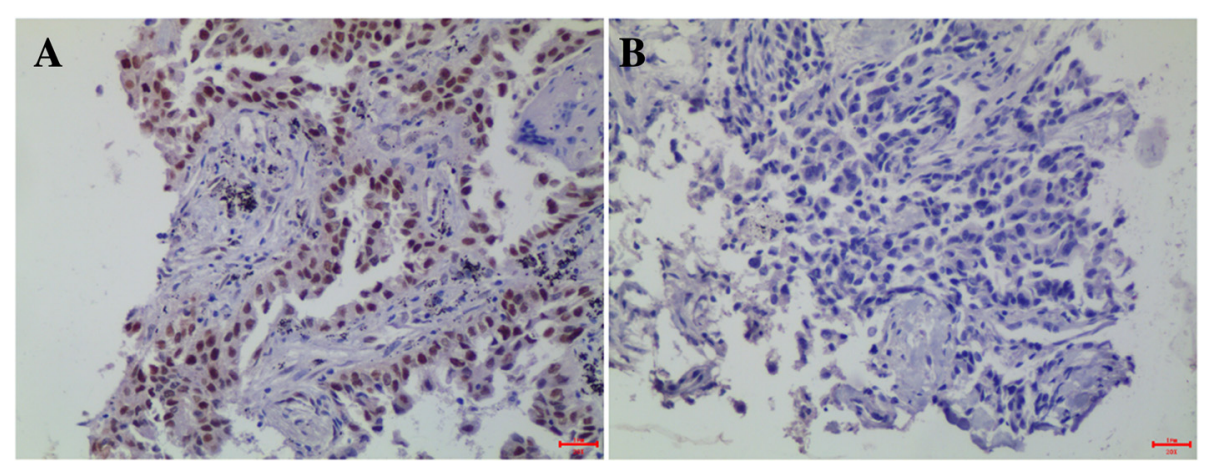

Figure 1 LSD1 expression in positive control and negtive control. (A) A lung adenocarcinomas that overexpression of LSD1 was as uesed as positive control. (B) "No primary antibody" including everything but the primary antibody showed negtive stain of LSD1 in the same lung adenocarcinomas tissue sample. 


\section{Results}

Expression of LSD1 in ovarian epithelial lesions

A lung adenocarcinomas that overexpression of LSD1 was as uesed as positive control as shown in Figure 1A. "No primary antibody" control showed negative stain of
LSD1 in the same lung adenocarcinomas tissue sample (Figure 1B). IHC staining revealed that LSD1 was localized mainly to the cell nucleus (dark brown nuclei) in different ovarian epithelial lesions (Figures 2 and 3). Positive IHC staining of LSD1 was observed in $50 \%$ of normal ovarian
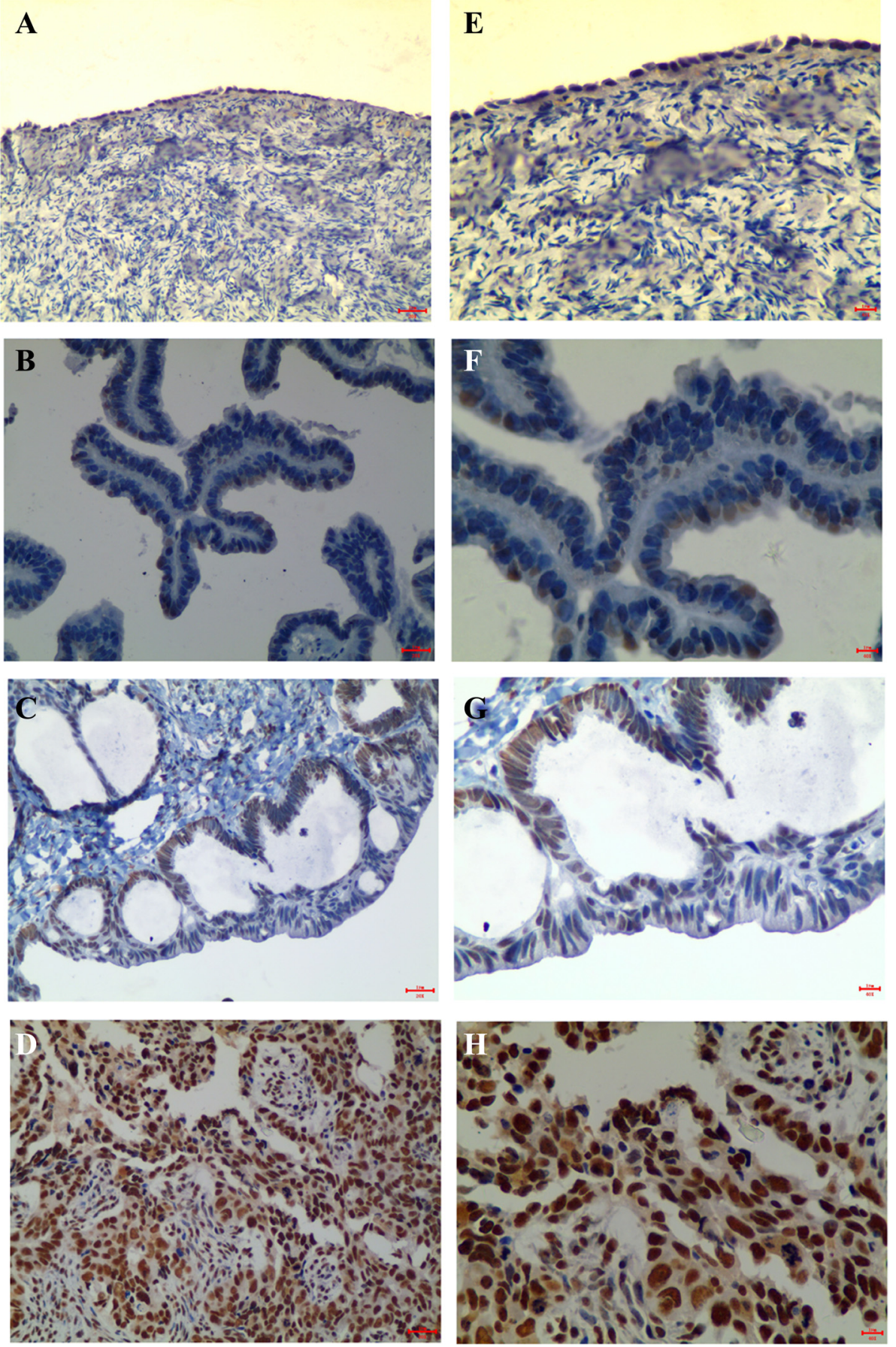

Figure $\mathbf{2}$ LSD1 expression and localization in (A) normal ovarian epithelia, (B) serous cystadenoma, (C) borderline serous cystadenoma, and (D) serous cystadenocarcinoma $(20 \times$, bar $=100 \mu \mathrm{m})$. The Higher magnification $(40 \times$, bar $=1 \mu \mathrm{m})$ was shown as the right image $(E, G, F, H)$. 

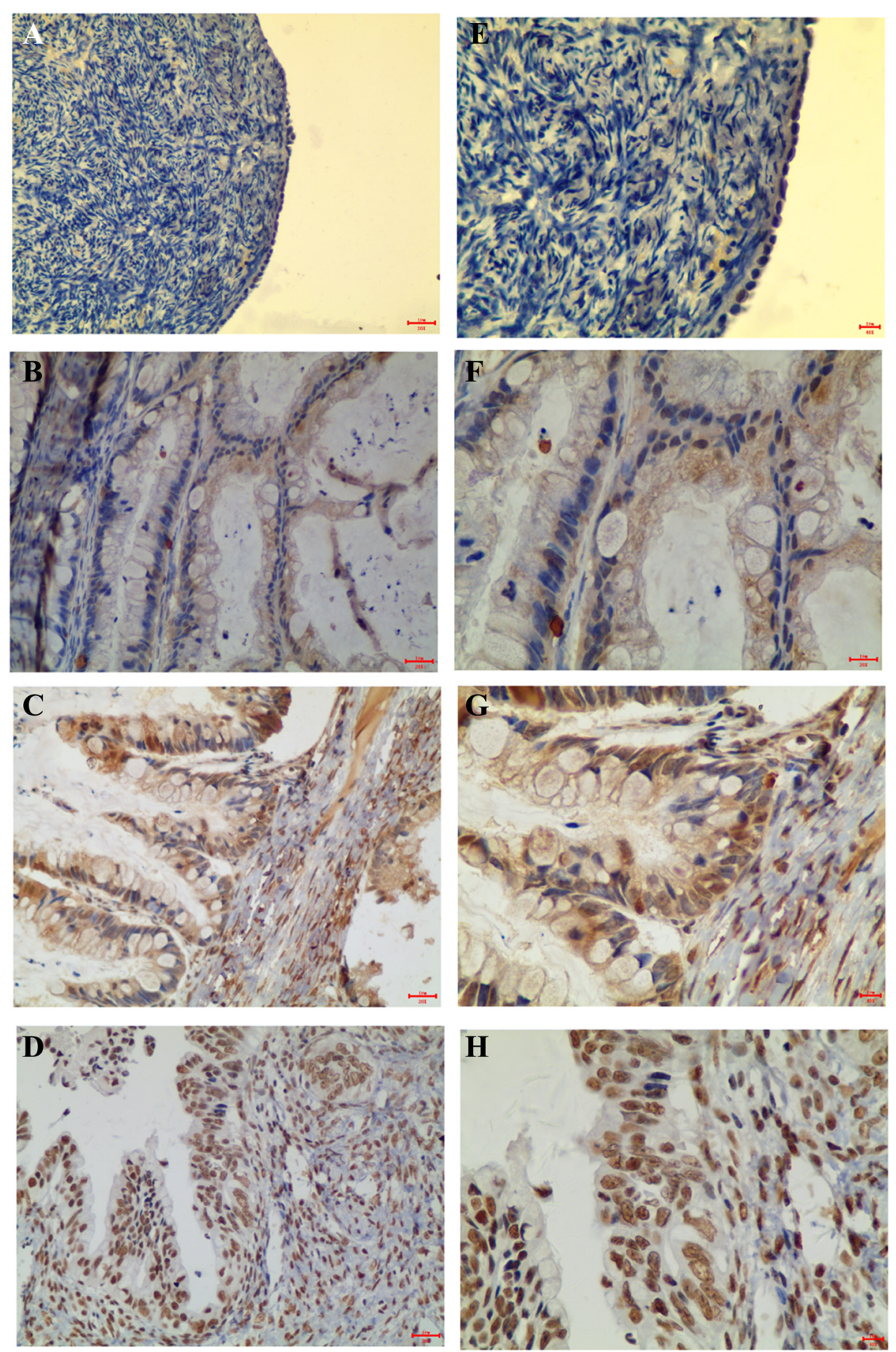

Figure $\mathbf{3}$ LSD1 expression and localization in (A) normal ovarian epithelia, (B) mucinous cystadenoma, (C) borderline mucinous cystadenoma, and (D) mucinous cystadenocarcinoma $(20 \times$, bar $=100 \mu \mathrm{m})$. The Higher magnification $(40 \times$, bar $=1 \mu \mathrm{m})$ was shown as the right image $(\mathbf{E}, \mathbf{G}, \mathbf{F}, \mathbf{H})$.

epithelia, $72 \%$ of serous cystadenoma, $73 \%$ of mucinous cystadenoma, $82 \%$ of borderline serous and mucinous cystadenoma, $94 \%$ of serous cystadenocarcinoma, and 92\% of mucinous cystadenocarcinoma, respectively (Table 1). LSD1 expression was significant statistical difference between the normal ovarian epithelia and all of carcinomas (benign, borderline tumors and carcinomas; $\mathrm{P}<0.001$ ). In additional, the incidence of LSD1 detected in epithelial carcinomas was significantly higher than that in benign and borderline tumors, both in subtypes of serous and 
Table 1 Expression of LSD1 in different ovarian epithelial lesions and normal ovarian epithelia

\begin{tabular}{|c|c|c|c|c|c|c|c|c|}
\hline \multirow[t]{2}{*}{ Clinicopathological features } & & \multirow[t]{2}{*}{ Cases $(\mathrm{N})$} & \multicolumn{4}{|c|}{ LSD1 } & \multirow[t]{2}{*}{ Positive (\%) } & \multirow[t]{2}{*}{$\mathbf{P}$} \\
\hline & & & - & + & ++ & +++ & & \\
\hline Normal ovarian epithelia & & 50 & 25 & 20 & 5 & 0 & 50 & $0.000^{\mathrm{ab}}$ \\
\hline \multirow[t]{4}{*}{ Serous epithelial lesions } & Serous cystadenoma & 53 & 15 & 25 & 10 & 3 & 72 & $0.010^{c}$ \\
\hline & Borderline serous cystadenoma & 50 & 9 & 10 & 25 & 6 & 82 & $0.000^{d}$ \\
\hline & & & & & & & & $0.002^{i}$ \\
\hline & Serous cystadenocarcinoma & 96 & 6 & 9 & 31 & 50 & 94 & $0.000^{\mathrm{ejk}}$ \\
\hline \multirow[t]{4}{*}{ Mucinous epithelial lesions } & Mucinous cystadenoma & 59 & 16 & 25 & 16 & 2 & 73 & $0.003^{f}$ \\
\hline & Borderline mucinous cystadenoma & 63 & 11 & 17 & 29 & 6 & 83 & $0.000^{9}$ \\
\hline & & & & & & & & $0.009^{1}$ \\
\hline & Mucinous cystadenocarcinoma & 36 & 3 & 5 & 9 & 19 & 92 & $0.000^{\mathrm{hmn}}$ \\
\hline
\end{tabular}

${ }^{a}$ Non-parametric Jonckheere-Terpstra test, $P$ value of normal and serous groups.

${ }^{\mathrm{b}}$ Non-parametric Jonckheere-Terpstra test, $\mathrm{P}$ value of normal and mucinous groups.

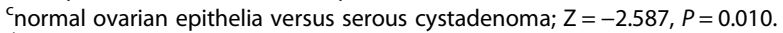

${ }^{d}$ normal ovarian epithelia versus borderline serous cystadenoma; $Z=-5.068, P=0.000$.

enormal ovarian epithelia versus serous cystadenocarcinoma; $Z=-8.404, P=0.000$.

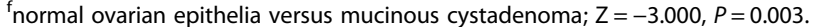

${ }^{g}$ normal ovarian epithelia versus borderline mucinous cystadenoma; $Z=-5.073, P=0.000$.

${ }^{h}$ normal ovarian epithelia versus mucinous cystadenocarcinoma; $Z=-6.277, P=0.000$.

'serous cystadenoma versus borderline serous cystadenoma; $Z=-3.126, P=0.002$.

${ }^{j}$ serous cystadenoma versus serous cystadenocarcinoma; $Z=-7.106, P=0.000$.

${ }^{k}$ borderline serous cystadenoma versus serous cystadenocarcinoma; $Z=-4.735, P=0.000$.

Imucinous cystadenoma versus borderline mucinous cystadenoma; $Z=-2.611, P=0.009$.

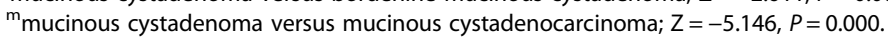

nborderline mucinous cystadenoma versus mucinous cystadenocarcinoma; $Z=-3.824, P=0.000$.

Z= Z-score.

mucinous $(\mathrm{P}<0.001)$. The incidence of LSD1 detected was gradually increased from benign and borderline to malignant ovarian tumors, suggusting that LSD1 protein was up-regulated in the development of serous or mucinous ovarian epithelial carcinoma.

\section{Association of LSD1 expression with clinicopathological features of EOC patient}

The correlation between LSD1 expression in ovarian carcinomas detected by IHC and clinicopathological features was further analyzed, including age, FIGO stage, tumor grade, lymphatic metastasis status and peritoneal cytology. The results showed that LSD1 expression was correlated with FIGO stage $(P=0.006)$ and lymph node metastasis $(P=0.001)$ among serous tumors (Table 2). Among mucinous tumors, significant association was also found between LSD1 expression and FIGO stage $(P=0.037)$ and lymph node metastasis $(P=0.026)$.

\section{Up-regulation of LSD1 is associated with poorer prognosis of EOC patient}

The potential correlation between expression of LSD1 and EOC prognosis was addressed in the present study. Patients were classified into low LSD1 expression group and high LSD1 expression group according to the IHC results. Kaplan-Meier curves suggested that overall survival time of patients with high LSD1 expression was significantly shorter than that of patients with low LSD1 expression $(P=0.0006$; Figure 4). Cox regression analyses were then conducted to analyze various prognostic parameters for survival of EOC patients. Univariate analysis identified three prognostic factors: FIGO stage (I, II, III, IV), lymphatic metastasis (negative, positive) and LSD1 expression (high, low). In multivariate Cox proportional hazard regression, LSD1 higher expression was a significant independent predictor of poor survival of EOC patients $(P=0.016)$, as well as FIGO stage $(P=0.019)$ (Table 3).

\section{Discussion}

Epigenetic alteration by histone methylation or demethylation has been shown to play an essential role in carcinogenesis and cancer progression. The methylation status of histone demethylases plays an important role in the regulation of gene expression [10]. Inhibition of chromatin modifying enzymes such as histone demethylases is a potential therapeutic strategy to inhibit cancer growth [11]. LSD1 had been found to control gene expression by histone modification. LSD1 is described as the first identified histone demethylase which represses and activates transcription by specifically demethylating the methyl groups from mono- and di-methylated Lysine (Lys) 4 of histone H3K4me and Lys9 of H3K9me [3,12]. Previous studies showed that LSD1 was aberrantly overexpressed in 
Table 2 Correlations between expression of LSD1 and clinicopathological features

\begin{tabular}{|c|c|c|c|c|c|}
\hline \multirow[t]{2}{*}{ Diagnosis } & \multirow[t]{2}{*}{ Characteristics } & \multirow[t]{2}{*}{ Cases (N) } & \multicolumn{2}{|c|}{ IHC results of LSD1 (N) } & \multirow[t]{2}{*}{$p$-value } \\
\hline & & & Low & High & \\
\hline \multirow[t]{18}{*}{ Serous cystadenocarcinoma } & Age & & & & 0.352 \\
\hline & $\leqq 60$ & 40 & 19 & 21 & \\
\hline & $>60$ & 56 & 30 & 26 & \\
\hline & FIGO stage & & & & 0.006 \\
\hline & । & 7 & 4 & 3 & \\
\hline & $\|$ & 23 & 5 & 18 & \\
\hline & III & 54 & 4 & 50 & \\
\hline & IV & 12 & 2 & 10 & \\
\hline & Tumor grade & & & & 0.707 \\
\hline & । & 30 & 6 & 24 & \\
\hline & $\|$ & 32 & 4 & 28 & \\
\hline & III & 34 & 5 & 29 & \\
\hline & Lymphatic metastasis & & & & 0.001 \\
\hline & Negative & 25 & 9 & 16 & \\
\hline & Positive & 71 & 6 & 65 & \\
\hline & Peritoneal cytology & & & & \\
\hline & Negative & 46 & 20 & 26 & 0.152 \\
\hline & Positive & 50 & 30 & 20 & \\
\hline \multirow[t]{18}{*}{ Mucinous cystadenocarcinoma } & Age & & & & 0.179 \\
\hline & $\leqq 60$ & 16 & 6 & 10 & \\
\hline & $>60$ & 20 & 13 & 7 & \\
\hline & FIGO stage & & & & 0.037 \\
\hline & I & 6 & 4 & 2 & \\
\hline & $\|$ & 9 & 3 & 6 & \\
\hline & III & 12 & 1 & 11 & \\
\hline & IV & 9 & 0 & 9 & \\
\hline & Tumor grade & & & & 0.995 \\
\hline & I & 9 & 2 & 7 & \\
\hline & $\|$ & 14 & 3 & 11 & \\
\hline & III & 13 & 3 & 10 & \\
\hline & Lymphatic metastasis & & & & 0.026 \\
\hline & Negative & 11 & 5 & 6 & \\
\hline & Positive & 25 & 3 & 22 & \\
\hline & Peritoneal cytology & & & & 0.311 \\
\hline & Negative & 15 & 9 & 6 & \\
\hline & Positive & 21 & 8 & 13 & \\
\hline
\end{tabular}

various kinds of cancer and higher expression of LSD1 associated with more aggression in breast cancer, prostate cancer, and lung cancer [5-7].

Only one study showed that overexpression of LSD1 mRNA in stage IIIC and high-grade ovarian tumors with the likely exception of mucinous tumors [9]. The higher levels of LSD1 mRNA was observed in serous, papillary serous, endometrioid, and clear cell tumors, but not in those of the mucinous subtype. The mucinous group in that cohort they studied consists of only a small number of tumors $(n=5)$. In our study, IHC results showed that higher expression of LSD1 protein in both serous 


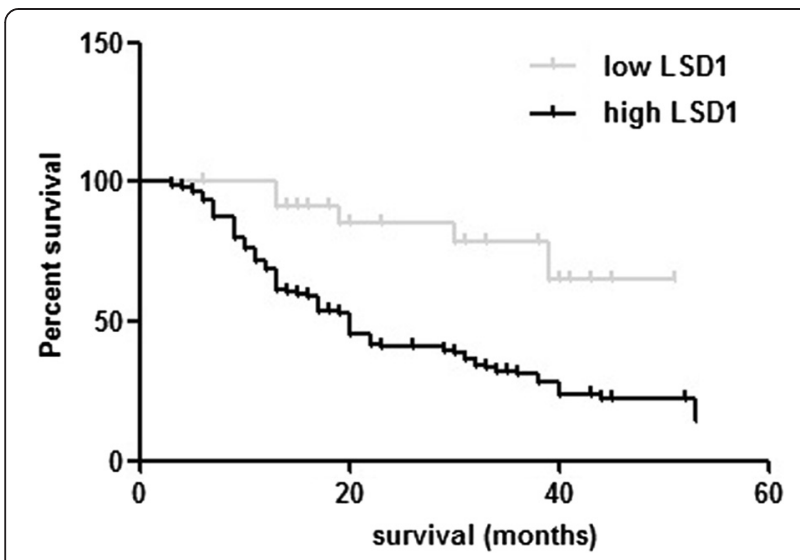

Figure 4 Overexpression of LSD1 predicts poor clinical outcome of EOC. Kaplan-Meier curves for overall survival according to expression of LSD1 in 132 EOC patients. (Log rank test, $P=0.0006)$

and mucinous cystadenocarcinoma compared to normal ovarian tissue. We think this inconsistence is due to realtive larger number of mucinous cystadenocarcinoma $(\mathrm{n}=36)$ detected in our study. In additional, diffrennt detection methods also contribute to the inconsistent results. Our results also showed that LSD1expression was increased with advanced FIGO stage, but not with tumor grade. High LSD1 expression was also associated with advanced tumor stage of pancreatic cancer but not tumor grade [13]. Our results indicated that high LSD1 expression was associated with more aggressive biological behavior. In fact, our results showed that LSD1 expression was correlated with lymph node metastasis among ovian tumors. There was also significant statistical difference of LSD1 protein expression between benign tumor groups and the normal groups. Therefore, LSD1 is potential to be an early diagnostic marker for serous or mucinous cystadenocarcinoma.

Furthermore, we found that the expression of LSD1 was gradually increased from benign and borderline tumors to epithelial carcinomas in a stepwise manner, both in subtypes of serous and mucinous, suggesting that LSD1 protein was up-regulated in the development of serous and mucinous ovarian epithelial carcinoma. So upregulation of LSD1 may be an early tumor promoting event in EOC. Previous reports also supported that LSD1 acts as an early tumor promoter in carcinogenesis through chromatin regulation [11,14-16]. LSD1 could repress p53 activity through demethylation of Lys370 in p53, thus inhibit p53mediated apoptosis and contribute to carcinogenesis [14]. Upregulation of LSD1 has also been considered as an early tumor promoting event in breast carcinoma [15]. There is a gradual increase of LSD1 expression within tumor progression from pre-invasive ductal carcinoma in situ to invasive ductal breast carcinoma [15]. Interruption of LSD1 by using siRNA or chemical inhibitor could suppress cancer cell proliferation, migration and invasion in various cancers [7,17-20] LSD1 knockout colorectal cancer cells showed less tumorigenic both in vivo and in vitro [21]. Chemical LSD1 inhibition could cause cytotoxicity in ovarian cancer lines [9]. Thus, our results indicate that LSD1 may be involved in the carcinogenesis and progression of EOC. The concrete mechanism in which LSD1 is linked to EOC development is deserved to been further explored.

IHC results also showed that higher expression LSD1 was associated with FIGO stage or lymphatic metastasis in both ovarian serous cystadenocarcinoma and mucinous cystadenocarcinoma. Of note, we found that EOC patients with high LSD1 expression had shorter overall survival time than patients with low LSD1 expression. Importantly, the Cox regression analyses identified LSD1 expression as a novel predicting factor for overall survival of EOC patients. It indicates that LSD1 expression may serve as a novel prognostic marker for EOC patients, and high LSD1 expression may promote tumor metastasis and associate with poor survival in ovarian epithelial carcinoma patients.

In conclusion, our results indicate that LSD1 is involved in carcinogenesis and progression in EOC. LSD1 may be an early identification and prognosis marker of EOC with promising therapeutic potential.

Table 3 Univariate and multivariate Cox regression analyses of clinicopathologic characteristics for overall survival in EOC patients

\begin{tabular}{llllll}
\hline Characteristic & & Univariate analysis HR $\mathbf{( 9 5 \% ~ C l )}$ & $\boldsymbol{P}$ value & Multivariate analysis HR (95\% Cl) & $\boldsymbol{P}$ value \\
\hline Age $(\mathrm{y})$ & $\leq 60,>60$ & $2.661(0.930-5.168)$ & 0.169 & & \\
FIGO stage & I, II, III, IV & $2.168(1.291-3.686)$ & $0.001^{* *}$ & $2.265(0.919-5.899)$ & $0.019^{*}$ \\
Lymphatic metastasis & negative, positive & $2.196(1.332-5.926)$ & $0.016^{*}$ & $0.818(0.136-3.219)$ \\
Peritoneal cytology & negative, positive & $1.566(0.566-5.861)$ & 0.519 & & \\
Tumor grade & I, II, III & $1.265(0.661-2.256)$ & 0.556 & & \\
LSD1 expression & low, high & $2.233(1.129-5.688)$ & $0.010^{*}$ & $2.808(1.131-6.967)$ & $0.016^{*}$ \\
\hline
\end{tabular}

$\mathrm{HR}$, Hazard ratio; $\mathrm{Cl}$, Confidence interval. Statistical significance. ${ }^{*} P<0.05 ;{ }^{*} P<0.01$. 


\section{Abbreviations}

LSD1: Lysine-specific demethylase 1; EOC: Epithelial ovarian cancer; IHC: Immunohistochemical.

\section{Competing interests}

The authors declare that they have no competing interests.

\section{Authors' contributions}

CY and XF conceived and designed the experiments. CC, GP and CY performed the experiments. QL analyzed the data and helped to draft the manuscript. CC and JG participated in the design of the study, performed the statistical analysis and drafted the manuscript. All authors read and approved the final manuscript.

\section{Acknowledgements}

This study was supported by the National Natural Science Foundation of China (No. 81101580)

\section{Author details}

${ }^{1}$ Department of Gynecology of Traditional Chinese Medicine, Jiangsu Provincial Hospital of Traditional Chinese Medicine Affiliated to Nanjing University of Traditional Chinese Medicine, Nanjing, Jiangsu 210029, China. ${ }^{2}$ Department of Endocrinology, Jiangsu Provincial Hospital of Traditional Chinese Medicine Affiliated to Nanjing University of Traditional Chinese Medicine, Nanjing, Jiangsu Province 210029, China. ${ }^{3}$ Department of Pathology, First Affiliated Hospital of Nanjing Medical University, Nanjing, Jiangsu 210029, China. ${ }^{4}$ Department of Medical Oncology, Second Affiliated Hospital, Zhejiang University College of Medicine, Hangzhou, Zhejiang 310009, China

Received: 8 January 2015 Accepted: 29 April 2015

Published online: 09 May 2015

\section{References}

1. Siegel R, Naishadham D, Jemal A. Cancer statistics, 2013. CA Cancer J Clin. 2013:63:11-30.

2. Cho KR, Shih le M. Ovarian cancer. Annu Rev Pathol. 2009;4:287-313.

3. Shi Y, Lan F, Matson C, Mulligan P, Whetstine JR, Cole PA, et al. Histone demethylation mediated by the nuclear amine oxidase homolog LSD1. Cell. 2004;119:941-53.

4. Ding J, Zhang ZM, Xia Y, Liao GQ, Pan Y, Liu S, et al. LSD1-mediated epigenetic modification contributes to proliferation and metastasis of colon cancer. Br J Cancer. 2013:109:994-1003.

5. Derr RS, van Hoesel AQ, Benard A, Goossens-Beumer IJ, Sajet A, Dekker-Ensink NG, et al. High nuclear expression levels of histone-modifying enzymes LSD1, HDAC2 and SIRT1 in tumor cells correlate with decreased survival and increased relapse in breast cancer patients. BMC Cancer. 2014;14:604

6. Suikki HE, Kujala PM, Tammela TL, van Weerden WM, Vessella RL, Visakorpi $T$ Genetic alterations and changes in expression of histone demethylases in prostate cancer. Prostate. 2010;70:889-98.

7. LV T, Yuan D, Miao X, LV Y, Zhan P, Shen X, et al. Over-expression of LSD1 promotes proliferation, migration and invasion in non-small cell lung cancer. PLoS One. 2012;7:e35065.

8. Zhao ZK, Yu HF, Wang DR, Dong P, Chen L, Wu WG, et al. Overexpression of lysine specific demethylase 1 predicts worse prognosis in primary hepatocellular carcinoma patients. World J Gastroenterol. 2012;18:6651-6.

9. Konovalov S, Garcia-Bassets I. Analysis of the levels of lysine-specific demethylase 1 (LSD1) mRNA in human ovarian tumors and the effects of chemical LSD1 inhibitors in ovarian cancer cell lines. J Ovarian Res. 2013;6:75.

10. Ueda R, Suzuki T, Mino K, Tsumoto H, Nakagawa H, Hasegawa M, et al. Identification of cell-active lysine specific demethylase 1-selective inhibitors. J Am Chem Soc. 2009;131:17536-7.

11. Hayami S, Kelly JD, Cho HS, Yoshimatsu M, Unoki M, Tsunoda T, et al. Overexpression of LSD1 contributes to human carcinogenesis through chromatin regulation in various cancers. Int J Cancer. 2011;128:574-86.

12. Metzger E, Wissmann M, Yin N, Muller JM, Schneider R, Peters AH, et al. LSD1 demethylates repressive histone marks to promote androgen-receptordependent transcription. Nature. 2005;437:436-9.
13. Qin Y, Zhu W, Xu W, Zhang B, Shi S, Ji S, et al. LSD1 sustains pancreatic cancer growth via maintaining HIF1alpha-dependent glycolytic process. Cancer Lett. 2014;347:225-32.

14. Huang J, Sengupta R, Espejo AB, Lee MG, Dorsey JA, Richter M, et al. p53 is regulated by the lysine demethylase LSD1. Nature. 2007:449:105-8.

15. Serce N, Gnatzy A, Steiner S, Lorenzen H, Kirfel J, Buettner R. Elevated expression of LSD1 (Lysine-specific demethylase 1) during tumour progression from pre-invasive to invasive ductal carcinoma of the breast. BMC Clin Path. 2012;12:13.

16. Bradley C, van der Meer R, Roodi N, Yan H, Chandrasekharan MB, Sun ZW, et al. Carcinogen-induced histone alteration in normal human mammary epithelial cells. Carcinogenesis. 2007;28:2184-92.

17. Willmann D, Lim S, Wetzel S, Metzger E, Jandausch A, Wilk W, et al. Impairment of prostate cancer cell growth by a selective and reversible lysine-specific demethylase 1 inhibitor. Int J Cancer. 2012;131:2704-9.

18. Hsu HC, Liu YS, Tseng KC, Yang TS, Yeh CY, You JF, et al. CBB1003, a lysine-specific demethylase 1 inhibitor, suppresses colorectal cancer cells growth through down-regulation of leucine-rich repeat-containing G-protein-coupled receptor 5 expression. J Cancer Res Clin Oncol. 2015;141:11-21.

19. Jin L, Hanigan CL, Wu Y, Wang W, Park BH, Woster PM, et al. Loss of LSD1 (lysine-specific demethylase 1) suppresses growth and alters gene expression of human colon cancer cells in a p53- and DNMT1(DNA methyltransferase 1)independent manner. Biochem J. 2013:449:459-68.

20. Ferrari-Amorotti G, Fragliasso V, Esteki R, Prudente Z, Soliera AR, Cattelani S, et al. Inhibiting interactions of lysine demethylase LSD1 with snail/slug blocks cancer cell invasion. Cancer Res. 2013;73:235-45.

21. Huang Z, Li S, Song W, Li X, Li Q, Zhang Z, et al. Lysine-specific demethylase 1 (LSD1/KDM1A) contributes to colorectal tumorigenesis via activation of the Wnt/beta-catenin pathway by down-regulating Dickkopf-1 (DKK1) [corrected]. PLoS One. 2013;8:e70077.

\section{Submit your next manuscript to BioMed Central and take full advantage of:}

- Convenient online submission

- Thorough peer review

- No space constraints or color figure charges

- Immediate publication on acceptance

- Inclusion in PubMed, CAS, Scopus and Google Scholar

- Research which is freely available for redistribution

Submit your manuscript at www.biomedcentral.com/submit
C) BioMed Central 